\title{
BOFdat: generating biomass objective function stoi- chiometric coefficients from experimental data
}

\author{
Jean-Christophe Lachance1, Jonathan M. Monk², Colton J. Lloyd², Yara Seif², Bernhard \\ O. Palsson ${ }^{2,3,4,5}$, Sébastien Rodrigue ${ }^{1,6}$, Adam M. Feist ${ }^{2,5}$, Zachary A. King ${ }^{2,}$ and Pierre- \\ Étienne Jacques ${ }^{1,6,{ }^{*}}$
}

1Département de Biologie, Université de Sherbrooke, Sherbrooke, Québec, Canada, ²Department of Bioengineering, University of California, San Diego, La Jolla, USA, ${ }^{3}$ Bioinformatics and Systems Biology Program, University of California, San Diego, La Jolla, USA, ${ }^{4}$ Department of Pediatrics, University of California, San Diego, La Jolla, CA, USA, ${ }^{5}$ Novo Nordisk Foundation Center for Biosustainability, Technical University of Denmark, Kongens Lyngby, Denmark, ${ }^{6}$ Centre de recherche du CHUS, Université de Sherbrooke, Sherbrooke, Québec, Canada

*To whom correspondence should be addressed.

\begin{abstract}
Genome-scale models (GEMs) rely on a biomass objective function (BOF) to predict phenotype from genotype. Here we present BOFdat, a Python package that offers functions to generate biomass objective function stoichiometric coefficients (BOFsc) from macromolecular cell composition and relative abundances of macromolecules obtained from omic datasets. Growthassociated and non-growth associated maintenance (GAM and NGAM) costs can also be calculated by BOFdat.

BOFdat is freely available on the Python Package Index (pip install BOFdat). The source code and an example usage (Jupyter Notebook and example files) are available on GitHub (https://github.com/jclachance/BOFdat). The documentation and API are available through ReadTheDocs (https://bofdat.readthedocs.io).

Contact: jean-christophe.lachance@usherbrooke.ca, zaking@eng.ucsd.edu, pierreetienne.jacques@usherbrooke.ca
\end{abstract}

Keywords: systems biology, mathematical modeling, metabolism, data integration

\section{Introduction}

Genome-scale models (GEMs) can be used to predict achievable physio-logical states given the constraints imposed by a metabolic network (O'Brien et al., 2015). When the GEM's objective is to maximize growth, the biomass objective function (BOF) is used to represent a reaction comprising all constituents of the organism (Feist and Palsson, 2010). The metabolites present in the BOF and their coefficients should ideally be obtained from experimental measurements performed in the desired conditions in order to better reflect the organism's growth requirements (Feist and Palsson, 2010; O'Brien et al., 2015). Despite its importance, the BOF is often mistreated when building GEMs (Xavier et al., 2017; Chan et al., 2017). The widely used Constraint-Based Recon-struction and Analysis toolbox for building metabolic models in Python, COBRApy (Ebrahim et al., 2013), does not include any functionality to incorporate experimental data into a BOF. Here we introduce BOFdat, a COBRApy compatible Python package that facilitates the integration of experimental data for the determination of the BOF.

\section{Implementation and features}

Biomass objective function stoichiometric coefficients (BOFsc) for five macromolecular categories (DNA, RNA, proteins, lipids and metab-olites) can be generated with BOFdat. Accurate calculation of BOFsc by BOFdat is performed as described in Thiele and Palsson, 2010 (Thiele and Palsson, 2010) using both the macromolecular weight fractions and the relative abundance of metabolites from omic datasets (Fig. 1). The architecture of the package is designed so that the BOFsc for each macromolecular category is generated independently using the appropriate generate_coefficients () function (Fig. S1). The resulting dictionary is then used with the corresponding update_biomass () function to update the BOF of the model. The parameters for these functions - thoroughly de- 
bioRxiv preprint doi: https://doi.org/10.1101/243881; this version posted January 5, 2018. The copyright holder for this preprint (which was not certified by peer review) is the author/funder, who has granted bioRxiv a license to display the preprint in perpetuity. It is made available under aCC-BY-NC 4.0 International license.

\section{J.-C.Lachance et al.}

scribed in the documentation (bofdat.readthedocs.io) - are mainly the sequence file of the organism, the macromolecular weight fraction of the category, and a comma-separated file containing the relative abundance of each molecule composing that category (Fig. 1). For metabolites and lipids, functions are provided to compare the experimental data with the prediction from the GEM, which allows informed decisions about compounds to include in the BOF (Fig. S1). Finally, a function to calculate ATP maintenance cost is also provided in BOFdat, using input growth data from different carbon sources, along with uptake and secretion rates (Fig. S1).

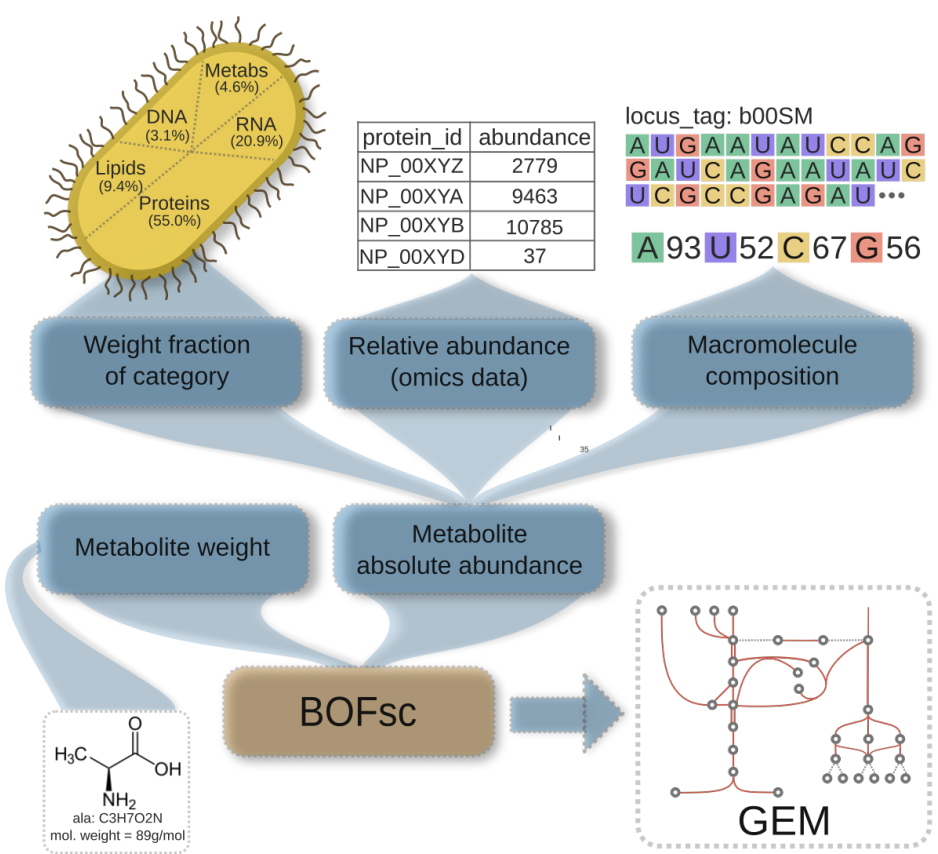

Fig. 1 - BOFdat overview. Metabolite absolute abundance is calculated based on the integration of the macromolecular category weight fraction (e.g. total protein weight per total cell weight), the relative abundance of each element composing a category (omics data), and the macromolecule composition (sequence). The weight and absolute abundance of each metabolite are in turn used to calculate BOFsc and to update the GEM.

\section{Usage and application}

To validate the use of BOFdat, available experimental omic datasets (Hayashi et al., 2006; Seo et al., 2014; Schmidt et al., 2016; Cheng et al., 2014; Oursel et al., 2007) were used to reconstruct the BOFsc of the high-quality iML1515 model of Escherichia coli K-12 MG1655 model (Monk et al., 2017; King et al., 2016), (see the example usage github.com/jclachance/BOFdat). BOFdat was able to generate BOFsc highly similar to those originally included in iML1515 (Fig. S2) based on experimental data only. When varying the weight fraction for each macromolecular category, the minimal error was found within $2 \%$ of the experimental weight fraction from the iML1515 BOFsc (Neidhardt et al., 1996; Feist et al., 2007; Monk et al. 2017). To further characterize the impact of the experimental data for BOFdat, the BOFsc of each amino acid were generated from 18 conditions that had both protein weight fractions and proteomic datasets from Schmidt et al., 2016. BOFsc for all samples were then compared to the condition in which cells were grown in presence of glucose, showing that BOFsc indeed varied across conditions $(8.74 \%+/-2.60 \%)$ and that the ones obtained in stationary conditions are the most different (Fig. S3A). We also found that the difference of protein weight fraction from the glucose growth condition was correlated with the average amino acid BOFsc difference (Pearson $r=0.959, \mathrm{p}$-value $=7.35 \mathrm{e}-12$ ) indicating that the most important parameter to generate BOFsc is the macromolecular weight fraction.

\section{Conclusion}

BOFdat enables the straightforward determination of BOFsc from mac-romolecular cell composition measurements and omic datasets. Using BOFdat in conjunction with precise inputs obtained from quantitative experimental data standardizes the procedure to generate BOFsc and provides a quality BOF, which is an essential component of 
bioRxiv preprint doi: https://doi.org/10.1101/243881; this version posted January 5, 2018. The copyright holder for this preprint (which was not certified by peer review) is the author/funder, who has granted bioRxiv a license to display the preprint in perpetuity. It is made available under aCC-BY-NC 4.0 International license.

BOFdat package

GEMs. BOFdat can be used for de novo BOF definition as well as to update pre-existing BOF when more accurate and potentially condition-specific data becomes available.

\section{Acknowledgements}

We thank Jared Broddrick for providing valuable information to determine GAM and NGAM, as well as Nathan Mih for helping with the Pypi distribution.

\section{Funding}

This work was supported by Natural Sciences and Engineering Research Council of Canada (NSERC) [to P.É.J. and S.R.], Fonds de recherche du Québec - Nature et technologies (FRQNT) [206064 to S.R.], Université de Sherbrooke, and by the Novo Nordisk Foundation through the Center for Biosustainability at the Technical University of Denmark [NNF10CC1016517 to B.O.P.].

Conflict of Interest: none declared.

\section{References}

Chan,S.H.J. et al. (2017) Standardizing biomass reactions and ensuring complete mass balance in genome-scale metabolic models. Bioinformatics, 33, 3603-3609.

Cheng,K.-K. et al. (2014) Global metabolic network reorganization by adaptive mutations allows fast growth of Escherichia coli on glycerol. Nat. Commun., 5, 3233.

Ebrahim,A. et al. (2013) COBRApy: COnstraints-Based Reconstruction and Analysis for Python. BMC Syst. Biol., 7, 74.

Feist,A.M. et al. (2007) A genome-scale metabolic reconstruction for Escherichia coli K-12 MG1655 that accounts for 1260 ORFs and thermodynamic information. Mol. Syst. Biol., 3, 121.

Feist,A.M. and Palsson,B.O. (2010) The biomass objective function. Curr. Opin. Microbiol., 13, 344-349.

Hayashi,K. et al. (2006) Highly accurate genome sequences of Escherichia coli K-12 strains MG1655 and W3110. Mol. Syst. Biol., 2, 2006.0007.

King,Z.A. et al. (2016) BiGG Models: A platform for integrating, standardizing and sharing genome-scale models. Nucleic Acids Res., 44, D515-22.

Monk,J.M. et al. (2017) iML1515, a knowledgebase that computes Escherichia coli traits. Nat. Biotechnol., 35, 904-908.

Neidhardt,F.C. et al. (1996) Escherichia coli and Salmonella: cellular and molecular biology Roy Curtis III, John L. Ingraham, Edmund C.C. Lin, K.Brooks Low, Boris Magasanik, William S. Reznikoff, Monica Riley, Moselio Schaechter, and H. Edwin Umbarger (ed) ASM Press, 1325 Massachusetts Ave., N.W., Washington, DC 20005.

O’Brien,E.J. et al. (2015) Using Genome-scale Models to Predict Biological Capabilities. Cell, 161, 971-987.

Oursel,D. et al. (2007) Identification and relative quantification of fatty acids in Escherichia coli membranes by gas chromatography/mass spectrometry. Rapid Commun. Mass Spectrom., 21, 3229-3233.

Schmidt,A. et al. (2016) The quantitative and condition-dependent Escherichia coli proteome. Nat. Biotechnol., 34, $104-110$.

Seo,S.W. et al. (2014) Deciphering Fur transcriptional regulatory network highlights its complex role beyond iron metabolism in Escherichia coli. Nat. Commun., 5, 4910.

Thiele,I. and Palsson,B.Ø. (2010) A protocol for generating a high-quality genome-scale metabolic reconstruction. Nat. Protoc., 5, 93-121.

Xavier,J.C. et al. (2017) Integration of Biomass Formulations of Genome-Scale Metabolic Models with Experimental Data Reveals Universally Essential Cofactors in Prokaryotes. Metab. Eng., 39, 200-208. 